\title{
LOCAL THRESHOLDING OF EYE FUNDUS IMAGES FOR EASY RETINAL VESSEL SEPARATION
}

\section{Mohammad Sami ${ }^{1}$, Dr. Avinash Gour ${ }^{2}$}

${ }^{1}$ Research Scholar, Dept. of Electronics and Communication Engineering, Sri Satya Sai University of Technology \& Medical Sciences, Sehore, Bhopal-Indore Road, Madhya Pradesh, India.

${ }^{2}$ Research Guide, Dept. of Electronics and Communication Engineering, Sri Satya Sai University of Technology \& Medical Sciences, Sehore, Bhopal Indore Road, Madhya Pradesh, India

Email: samisbit@gmail.com

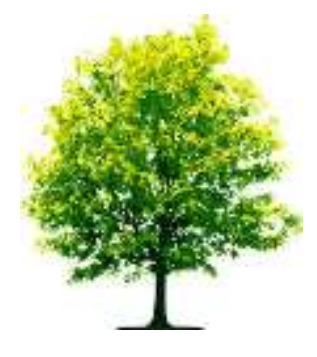

Keywords:

Diabetic Retinopathy, Fundus Image, Segmentation approach, Pre-processing,

EFI images.

\section{A B S T R A C T}

Early diagnosis is required for diabetic retinopathy. It may prevent the disease from further complications. It is divided into one of two phases (early and final stages of non-proliferation and proliferative respectively). In this paper, our experiments and conclusions while applying an effective segmentation approach with two phases, together with structure extraction filters, as preprocessing steps to detect the individual lesions such as microaneurysms, haemorrhages or exudates and segmentation is demanding due to the highly variable luminance, color and definition of the EFI images. We have included experimentation results that test runtime, fraction of lesions segmented and accuracy of the detection phase. 


\section{International Journal of}

Advanced Multidisciplinary Scientific Research (IJAMSR) ISSN:2581-4281

\section{Introduction}

The effects of diabetes on the eye are diabetic retinopathy (DR). Diabetic retinopathy (DR) is associated with microvascular changes in the retina causing diabetes, which leads to further blindness. It mainly affects people between the ages of 20 and 60. According to the study, diabetic retinopathy (DR) is divided into two phases. Non-proliferative and proliferative. Non-proliferative is known as early stage and proliferative is known as the last stage of diagnosis. Non-proliferative diabetic retinopathy (NPDR): Another name for NPDR is background retinopathy. The eye does not form new blood vessels as the initial phase of diabetes. This is called non-proliferative. During the initial phase, blood and fluid leak in the damaged blood vessels of the eye. Often, the retina in the center starts to swell. This condition is often called macular edema. Again, there are three phases of NPDR with mild, moderate and severe non-proliferative retinopathy. The first manifestation of NP-DR is microaneurysms, then bleeding and exudate. Exudates are composed of proteins and lipids that pass through the walls of vessels and are deposited in the retina. They can be divided into two types: soft and solid exudate. They are characterized by yellowish color, round shape and alternating size/brightness, depending on the stage of the disease. The mild step is diabetic retinopathy of the earliest stage, enlarged capillaries, which resemble a small red dot or balloon similar to the swelling of the retinal blood vessels. They are known as microaneurysm and these vessels run into the eyes. It does not affect eyesight. In a moderate stage, the blood vessels nourishes the retina and can even block. This results in diabetic macular oedema (DME), which builds fluid in the macular region. These changes often cause a visible change in the retina. In a serious stage the increase in the number of blood vessels in the retina is blocked, reducing the necessary blood report in the retina. The retina is marked for the cultivation of new blood vessels. Proliferative Diabetic retinopathy: This is the most accurate stage in diabetic retinopathy. At this stage, new blood vessels begin to grow in the retina and glassy gel. These blood vessels are usually weak and unusual in size and grow in the middle of the eye, which can lead to blood leakage in the eye, visual disturbances and blindness. 


\section{International Journal of \\ Advanced Multidisciplinary Scientific Research (IJAMSR) ISSN:2581-4281}

\section{Proposed System}

Density-based grouping method can be quite effective in segmenting the image. But individual pixels are too small and variable for the definition of macro-regions. In the twostep approach, firstly calculates super pixels from individual pixels. Superpixel is a (small) cell in a network of fully connected local regions [1]. The set of all pixels and the superpixel algorithm calculates and adjusts these to capture the homogeneity of the region. The term superpixel is derived from that groups the adjacent pixels in the larger (super) pixels. The calculation of the pixels local homogeneous regions are using strict criteria (a number of super pixels and maximum weighted of the colour and the spatial distance threshold). Subsequent density part of the procedure will bring together regions whose homogeneity is above a certain threshold.

Super pixels can be created using the simple linear iterative Clustering (SLIC) algorithm. SLIC is a K-means based approach called with a parameter that number of super pixels. The initial super pixels are equally good spread kmeans centroids of hexagonal cells with an equal area. The algorithm then iteratively evolves the cells using k-means, causing the original cells to be deformed into misshapen polygonal regions based on color homogeneity ${ }^{[2][3]}$. The end result is still a connected set of non-overlapping small "deformed" colour-homogeneous local regions that together whole picture. To improve color distance calculation, the CIELab color space is used instead of Rgb. CIELab color space was developed with the intention of to better mimic human perceptual colour differences than some other color spaces, such as RGB. The most important SLIC's 26parameters are the number of super pixels $\mathrm{K}$ and the radius $\mathrm{r}$. The radius determines that areas smaller than the merged with adjacent regions. The initial and average superpixel size is therefore $\mathrm{N} / \mathrm{K}$ where $\mathrm{N}$ is the number pixels in the image and $\mathrm{K}$ is the number of super pixels. Figure 1 shows the result of applying SLIC with $\mathrm{K}=3000$ on an EFI image. Since some lesions can be quite small (e.g. micro-aneurisms), it is advantageous to have a higher number of super pixels, as shown in Figure $2(\mathrm{~K}=15000)$, because it better able to isolate small structures. On the other hand SLIC is arithmetically heavy, and runtime is increasing with $\mathrm{K}$. 


\section{International Journal of Advanced Multidisciplinary Scientific Research (IJAMSR) ISSN:2581-4281}

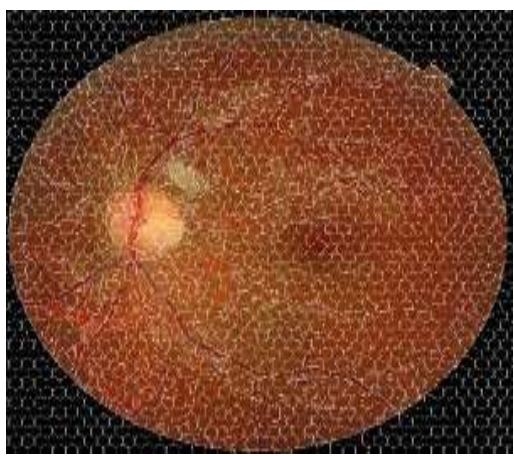

Figure 1 Input Image

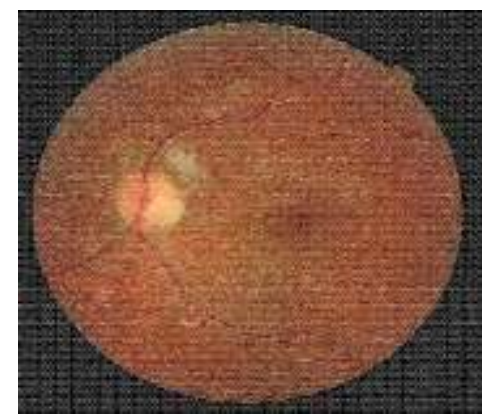

Figure 2 Input Image

The super-pixelized image of Figure 2 is ready for segmentation using density-based clustering, with DBScan [4][5]. DBScan is based on the classification of points into three different groups, based on density: core-points, border points and noise points. A super-pixel is classified as a core point iff it has at least a specified number of "similar" points (minPts) at a distance less than $r$ (radius). Transitivity ensures that if a point $p$ is a super-pixel of a region, then all super-pixels are "density accessible" from it based any chain of dense (minPts, r) connectivity will be added to the region. The fact that each point needs to be analyzed more than once in DBScan makes the algorithm slow. However, since it is applied to super-pixels, the process runs much faster than if ran against individual pixels. Figure 3 shows the result of applying DBscan with a specific threhsold. Given a set of super-pixels, DBScan groups those that are closest in terms of color and spatial properties. 


\section{International Journal of Advanced Multidisciplinary Scientific Research (IJAMSR) ISSN:2581-4281}

\section{Methodology}

Automated detection of diabetic retinopathy from the eye fundus has filmed the interest of researchers and practitioners. Works that are processed in advance, detection of lesions, extraction of characteristics and classification.

Pre-Processing: Pre-Processing/Pre-treatment eliminates unnecessary information and improves data for further analysis. According to him, the images are normalized against a reference model (an image chosen by efficient analysis[6][7]. The usual approach to dealing with variability distinguishes (Differences in light dissipation, presence of abnormalities and variation of reflection and thickness of the retina) and intervariability (different means/angles, acquisition, lighting and pigment of the retina). This step includes, among other things, that the brightness, of intensity, contrast and levelling of the histogram. Shadow correction is a preprocessing step based on the medium/middle filtering method. The image background (which contains all variations of lighting) is obtained by smoothing out the original image, the medium or medial filter is configured with a size that should be larger than the largest retinal structure. The correction of shadows reduces the sound in the image (shot noise and salt pepper). Morphological transformations, dilations and erosion are applied to binary images, relying on predetermined structural elements[8]. The structural elements interact with the geometric shapes in the image by changing them, receiving the relevant information. To emphasize the fine and welldefined contours, it is ideal to use a small structural element, because large structural elements do not find objects smaller than the size of the structural element itself. The histogram alignment divides the histograms for each color channel of the original image, so 
that the processed image contains a more even distribution of the pixel values. Alternatively, the histogram specification allows color normalization by interpolating the pixel values in each channel. This method makes the structures more distinguishable from each other and from the background. To improve local contrast, adaptive histogram levelling is used. Transformation "Radon" is applied using a small window centered on the candidate for lesion (in particular, a microaneurysm), providing information on the classification of the model. Adaptive threshold reporting takes into account brightness changes in the image and changes the thresholds from pixel to pixel. It consists of two successive steps: dividing images into homogeneous regions and targeting applicants for injuries with detection techniques and techniques to enlarge the region.

Detection of Lesions: For the detection of microaneurysms, first removal of the blood vessels from the image is necessary, in order to reduce false positives. This can be done easily by removing large objects using the transformation, morphological operators (Tophat transform), doubling filters or pixel classification. Since the optical disc characteristics of exudates (brightness and contour) must be identified in order to noninterference with their detection. The optical disc has a smooth texture and can be removed by the morphological operators and the watershed transformation and the algorithm "Otsu". This transformation of watershed used to identify the border of Optical disc based on variations of gray image levels, since this structure is a clear area and blood vessels that generates in dark, with very intensive variation. The level setting method is a method for controlling the optical disc which includes a first curve-level set of level 0- of larger area and develops this surface so that level set of level 0 is approached with the optical disc[9]. Morphological filtering is used to isolate areas related to exudates based on a linear classification built on color properties and contours of lesions. Structures that fulfill brightness and boundary eligibility conditions are classified as exudates. There are two methods in literature to detect hemorrhages namely, detection of blood vessels and detection of blood vessels with hemorrhages[10][11]. The two top-down and bottom-up methods were designed to lighting changes in the image. They use contrast algorithms with the growth of the region approaches to cutting out the candidate regions. Otsu algorithm is used, at the 
Vol 3, Issue 10, 2020 Imfact Factor:2.58 DOI: https://doi.org/10.31426/ijamsr.2020.3.10.3812

threshold of the image[12][13]. The values of the thresholds differ from the every image, depending on the choice automatically made by the algorithm. As a result, high-intensity pixels are reconstructed, while the rest are removed.

Classification: used to identify lesions of the certain regions or to determine a degree of severity of the disease from the entire image[14][15]. In the first case the characteristics are extracted from the candidate and training data regions with the correct class. Algorithm for building classifier use this set of training data. Ensemble of Naive base plus supporting vector machines (SVM) is used to reduce the number of characteristics iteratively, then a final set of features was submitted in the SVM classifier[16][17]. The classifier for SVM tends to contours of exudates with improved accuracy, often receives a small number of false positives. K-means grouping is used for the determination of two groups: Exudates and non-exudates. Initially, the maximum and minimum aid intensities are calculated and used as centres of clusters that are updated by the iteratively algorithm.

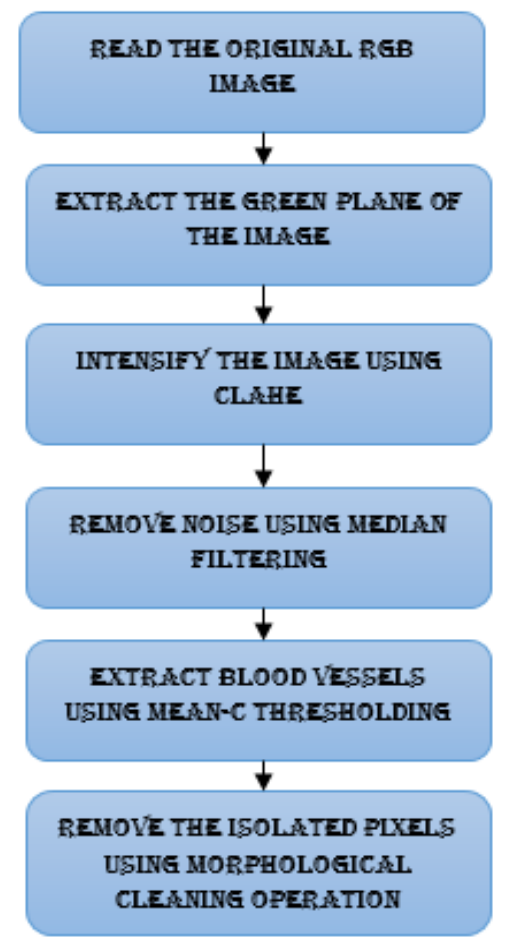

Figure 4: Flow Chart of the Proposed Model 
Vol 3, Issue 10, 2020 Imfact Factor:2.58 DOI: https://doi.org/10.31426/ijamsr.2020.3.10.3812

\section{Results}

MATLAB (matrix laboratory) is a highperformance language for technical computing. Math and computation, Algorithm development, Data acquisition, Data analysis, exploration, visualization, etc., Only technique applicable to this system is,

i) Threshold Method
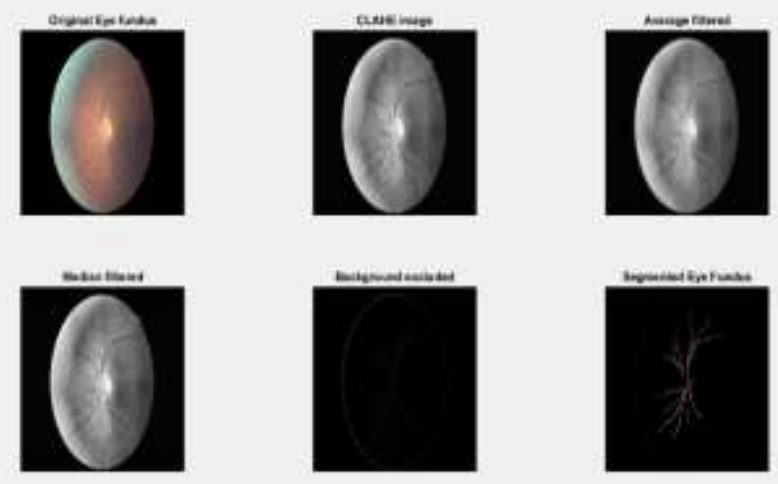

Figure 4: Output Image

\section{Conclusion}

Considering the results of the runtime and the classification accuracy of the SLIC and Dbscan of eye fundus images by Messidor database. The aim was to compare quality of the resulting segmentation using a series of statistics: Runtime is the time segmentation takes to perform compared to the number of super pixels. More super pixels will make it possible individualizing more lesions, but on the other hand will take more time to run. This runtime is confronted with the fraction of injuries individualised by segmentation, when compared to the highest detail reported (15000 super pixels). Finally, we also measure classification accuracy, to compare classification of Slic output with classification of Slic+DBScan. Segmented regions from 100 images were referred to as exudates, microaneurysm, bleeding or normal eye fundus, features were extracted. 
Vol 3, Issue 10, 2020 Imfact Factor:2.58 DOI: https://doi.org/10.31426/ijamsr.2020.3.10.3812

\section{References}

1) A. Imran, J. Li, Y. Pei, J. J. Yang, and Q. Wang, "Comparative analysis of vessel segmentation techniques in retinal images," IEEE Access, vol. 7, pp. 114862-114887, 2019.

2) Kolb, H. Simple Anatomy of the Retina. In Web vision: The Organization of the Retina and Visual System; Kolb, H., Fernandez, E., Nelson, R., Eds.; University of Utah Health Sciences Center: Salt Lake City, UT, USA, 1995; Available online: http://europepmc.org/books/NBK11533;jsessio nid $=4 C 8 B A D 63 F 75 E A D 49 C 21 B C 65 E 2 A E 5 F 6$ F3 (accessed on 22 January 2018).

3) H. A. Leopold, J. Orchard, J. S. Zelek, and V. Lakshminarayanan, "PixelBNN: augmenting the PixelCNN with batch normalization and the presentation of a fast architecture for retinal vessel segmentation," Journal of Imaging, vol. 5, no. 2, p. 26, 2019.

4) MESSIDOR: Methods for Evaluating Segmentation and Indexing Techniques Dedicated to Retinal Ophthalmology, 2004. Available

online: http://www.adcis.net/en/DownloadThird-Party/Messidor.html (accessed on 22 January 2018).

5) T. Chakraborti, D. K. Jha, A. S. Chowdhury, and X. Jiang, "Aself-adaptive matched filter for retinal blood vessel detection," Machine Vision and Applications PP 2014.
6) Kolb, H. Simple Anatomy of the Retina, 2012. Available

online: http://webvision.med.utah.edu/book/par $t$-i-foundations/simple-anatomy-of-theretina/ (accessed on 22 January 2018).

7) Soomro TA, Gao J, Khan T, Hani AFM, Khan MAU, Paul M. Computerized approaches for the detection of diabetic retinopathy using retinal fundus images: a survey. Pattern Analysis and Applications. 2017.

8) M.M. Fraz, A. Basit, S.A. Barman Application of morphological bit planes in retinal blood vessel extraction J Digit Imaging, 26 (2) (2013), pp. 274-286.

9) Jusoh $F$, Haron $H$, Ibrahim $R$, Azemin $M$ (2016) An overview of retinal blood vessels segmentation. Advanced computer and communication engineering technology. Springer, Berlin.

10) Kolb, H. Simple Anatomy of the Retina. In Web vision: The Organization of the Retina and Visual System; Kolb, H., Fernandez, E., Nelson, R., Eds.; University of Utah Health Sciences Center: Salt Lake City, UT, USA, 1995; Available

online: http://europepmc.org/books/NBK11533; jsessionid $=4 C 8 B A D 63 F 75 E A D 49 C 21 B C 65 E 2$ AE5F6F3 (accessed on 22 January 2018).

11) Akram, M.U., Khan, S.A.: Multilayered thresholding-based blood vessel seg-mentation for screening of diabetic retinopathy. Eng. Computer. 29, 165-173 (2013) 
Vol 3, Issue 10, 2020 Imfact Factor:2.58 DOI: https://doi.org/10.31426/ijamsr.2020.3.10.3812

12) Liskowski, P., Krawiec, K.: Segmenting retinal blood vessels with deep neural networks. IEEE Trans. Med. Imaging 35(11), 2369-2380 (2016)

13) . Fraz MM, Remagnino $P$, Hoppe $A$, Uyyanonvara B, Rudnicka AR, et al. (2012) Blood vessel segmentation methodologies in retinal images-a survey. Computer Methods Programs Biomed 108(1): 407-433.

14) Alberola, J.M.; del Val, E.; Costa, A.; Novais, P.; Julian, V. A genetic algorithm for group formation in elderly communities. AI Commun. 2018, 31, 409-425.

15) Chamoso, P.; De Paz, J.F.; De La Prieta, F.; Bajo Pérez, J. Agreement technologies applied to transmission towers maintenance. AI Commun. 2017, 30, 83-98.
16) E. Sorantin, C. Halmai, B. Erdohelyi, $K$. Palagyi, L. Nyul, K. Olle, B. Geiger, F. Lindbichler, G. Friedrich, and K. Kiesler, "Spiral-ct-based assessment of tracheal stenoses using 3-dskeletonization", IEEE Trans. on Med. Img., vol. 21, pp. 263-273, March 2002

17) Fraz MM, Remagnino $P$, Hoppe $A$, Uyyanonvara B, Rudnicka AR, et al. (2012) Blood vessel segmentation methodologies in retinal images-a survey. Comput Methods Programs Biomed 108(1): 407-433. 